\title{
MÁS INTELIGENTES DE LO QUE EL COLEGIO CREE
}

\author{
Maurizia D'Antoni ${ }^{1}$ \\ Xenia Pacheco Soto ${ }^{2}$
}

Las autoras plantean un ensayo sobre el concepto de inteligencia que existe, en térninos generales, en la cultura educativa costarricense, la cual es una visión tradicional, decimonónica y sin apego a la realidad que viven los y las estudiantes de la educación secundaria.

A partir de ahi, inician una exploración sobre los hallazgos que Goleman y Gardner han realizado sobre la inteligencia emocional y las inteligencias múltiples. Se plantea que la institución educativa aún no ha incorporado estaas últimas tendencias y es necesaria una renovación que le otorgue nuevos significados al acto de aprender.
The authors write about the prevailing concept of intelligence, basically in the Costa Rican educational system, which is an oldfashioned, traditional viewpoint that has no relation with the reality of high school students. Based on that information, they start to explore the findings of Goleman and Gardner on emotional intelligence and multiple intelligences. It is said that the educational centers have not incorporated these last tendencies and there is a need for a renovation that will assign new meanings to the act of learning.

1 Educadora y Psicóloga italiana. División de Educología, Universidad Nacional.

2 Geógrafa y Educadora. División de Educología y Escuela de Ciencias Geográficas. Universidad Nacional. 


\section{Introducción}

El concepto que los y las educadores (as) tengan sobre la inteligencia es central en su quehacer pedagógico, pues este participa en la definición y orientación de los objetivos que lo guían tanto como en la intencionalidad de su trabajo y, en consecuencia, impregna las estrategias didácticas mediante las cuales se propone lograr tales objetivos. Incluso, se expresa en las relaciones interpersonales que construyen, junto con otros factores, el clima para los aprendizajes en el aula. Reflexionar acerca de lo que es la inteligencia, en relación con los procesos de enseñanza - aprendizaje, especialmente en la enseñanza media, resulta entonces de gran importancia para una mejor comprensión de tales procesos y esto es lo que nos proponemos hacer en este artículo.

El artículo quiere ser una reflexión con el formato de un ensayo, un trabajo original de reflexión que se nutre de puntos de vista, propuestas y reflexiones de las autoras, así como de material investigativo, de las mismas autoras o de otros.

Para iniciar la reflexión, haremos un recorrido que nos permita revisar, de manera breve, las principales ideas que constituyen la base de los significados del concepto inteligencia. Recordemos que este término está conformado por otros dos que son eligere y el prefijo inter, los cuales significan en latín, respectivamente," escoger" y "entre", por lo que podemos deducir que la connotación fundamental del concepto inteligencia, se refiere a la capacidad para escoger, para tomar decisiones y elegir, frente a alternativas y situaciones diversas, la mejor. (Antunes, 1988).

No obstante lo anterior, es nuestra opinión que aún priva tanto en la cultura educativa costarricense en general, como en la enseñanza media en particular, el concepto tradicional de inteligencia, según el cual, casi solo es inteligente quien muestre buenas notas especialmente en matemática y en algunas de las llamadas ciencias exactas, como la química y la física. Esta idea propicia maneras particulares de ver el mundo y se corresponde, por lo general, con formas de pensamiento reduccionistas y fragmentadas. (En Costa Rica, véase D'Antoni y Pacheco, 2002. En el mundo: Bertolini, 2003 y Mc Laren, 1986).

Por suerte, las investigaciones sobre el tema de la inteligencia humana, han arrojado resultados que evidencian la reducción que se hace cuando se aplica el concepto de inteligencia, según el criterio tradicional, por cuanto abrevia, atomiza, disminuye y rebaja toda la complejidad y riqueza que entraña la inteligencia humana y en consecuencia, las múltiples y diversas formas de ver y construir el mundo, así como de aprender, enseñar y especialmente, de educar, como se verá a continuación.

Algunos de los ejemplos de la corriente que busca rescatar los múltiples significados y expresiones del concepto que se menciona, son los trabajos de Goleman (1995) sobre inteligencia emocional (IE) y de Howard Gardner (1996) 
En el marco tradicional en el que se define la inteligencia, se nos juzga por cuanto sabemos, por la rapidez con que captamos lo que el profesor está diciendo o lo que se quiere de nosotros, ojalá sin hacer muchas preguntas. Se valora nuestra capacidad para adaptamos a un sistema que ya existía cuando llegamos y que difícilmente podríamos cambiar. En ese sistema percibimos que nuestro éxito o fracaso depende en parte del estudio, pero también depende de características "innatas", que algunos tenemos y otros no tenemos. sobre inteligencias múltiples, (MI). Sus libros venden millones de ejemplares y son traducidos a las principales lenguas extranjeras. El nombre de los dos estudiosos empieza a hacerse famoso entre los entendidos y no entendidos del tema. El volumen de publicaciones y el entusiasmo que este provoca, responde tanto a la intención divulgativa de algunas publicaciones, según lo hace explícito, por ejemplo, el mismo Goleman, como también por la satisfacción que provoca en la gente, "descubrimientos" de esta índole.

$\mathrm{Al}$ respecto nos parece que ambos intentos tienen en común haber creado ambientes propicios para dudar del concepto tradicional de inteligencia, que re-

cibe con estos un severo revés, junto con el tema de la medición del CI o IQ, que se refiere al coeficiente de la inteligencia. Así, las teorías de dichos autores pueden interpretarse como una plataforma conceptual para la revisión crítica de la acepción radicional de inteligencia, tan arraigada en nuestras propias vivencias escolares y es probable que también nos permita sospechar acerca de sus relaciones con el currículum, explícito y, sobre todo, implícito u oculto, de la educación media y por supuesto con el quehacer en el aula, con el cual se corresponde.

Es probable que la aceptación de las nuevas teorías sobre las inteligencias, esté ligada al hecho de que la mayoría de las personas se reconoce más en la amplitud que ofrecen los nuevos postulados que en los tradicionales. Muchas personas, por ejemplo, recuerdan haber sido lastimadas en la escuela y el colegio, por la imagen que de sí mismas les arrojaba el resultado de alguna prueba de inteligencia, o bien exámenes de admisión, o soluciones de tareas más o menos cotidianas. Todo ello fundamentado en la concepción cuantitativa de la inteligencia, que supuestamente da la medida de lo que deberían ser para lograr el éxito, gustarle a las autoridades, o bien ser aprobadas por sus pares y bendecidas por la cultura.

Puede ser que inclusive a quienes les ha ido bien, o relativamente bien, les resulte estresante el modelo tradicional de inteligencia mediante el cual se les ha calificado así, y prefieran el modelo de inteligencia más amplio e inclusivo. No siempre el diez es alcanzable y la escuela frecuente y rebuscadamente, coloca obstáculos y trampas, para que este no se alcance, lo cual resulta especialmente 
grave para el desarrollo de las personas, si consideramos que la escuela y el colegio, son los primeros sitios públicos donde uno es juzgado y en donde probamos nuestras posibilidades frente a grupos de pares y autoridades. La IE describe un mundo en el que lo emocional y lo cognitivo, están articulados, y en el que cada uno de esos ámbitos requiere del otro para desarrollarse y crecer. En dichas instituciones educativas, por lo general, esta articulación no ha sido considerada en las tareas pedagógicas, sino que por el contrario, en ellas se privilegia lo intelectual por sobre lo emocional, con lo cual no solo se mantiene la separación de procesos que son integrados y de cuya mutua fecundación depende la calidad y significancia de los aprendizajes, sino que se asume un paradigma que supone que la exclusión de lo emocional es condición previa necesaria, para lograr la apropiación del conocimiento de manera "objetiva". Si buscamos en nuestra propia experiencia, afinando la mirada, es probable que podamos reconocer en nuestros procesos de aprendizaje, sentimientos de temor, alegría, miedo, vergüenza, satisfacción, inseguridad, entusiasmo, confianza, etc., más que acompañando tales procesos, formando parte integral de aquellos.

En el marco tradicional en el que se define la inteligencia, se nos juzga por cuanto sabemos, por la rapidez con que captamos lo que el profesor está diciendo o lo que se quiere de nosotros, ojalá sin hacer muchas preguntas. Se valora nuestra capacidad para adaptarnos a un sistema que ya existía cuando llegamos y que difícilmente podríamos cambiar. En ese sistema percibimos que nuestro éxito o fracaso depende en parte del estudio, pero también depende de características "innatas", que algunos tenemos y otros no tenemos. Así lo expresan, por ejemplo, los clásicos monólogos de los docentes hacia los padres y madres de familia, los cuales incluyen en su repertorio oraciones como "es inteligente, pero no estudia" o bien, "iqué especial que es su hija i" o "Mire, ya yo he hecho todo lo que he podido, pero! qué va!", él / ella no pone de su parte, y si sigue así...". Una investigación interesante incluiría grabar algunas de esas conversaciones y analizar su contenido, o bien buscar en los exalumnos, ya adultos, el rastro que tales opiniones han dejado en su visión de sí mismos o del mundo.

De cualquier forma, el tema de la inteligencia atemoriza y el tema de las inteligencias múltiples, de la inteligencia emocional, aplaca ansiedades, provoca identificación, interesa y emociona. De un lado, parece relajarnos el saber que podemos fallar o presentar debilidades en un área específica y sobresalir en otra, y que según los científicos investigadores de las inteligencias no existe la jerarquía de un área sobre otra.

Esa jerarquización evidentemente existió en el colegio, donde no era lo mismo ser muy buenos en educación física que en matemática, por citar dos asignaturas, con la intención de subrayar la contradicción y aclarar lo mencionado. 
Nos alivia y nos gusta que en el contexto conceptual de la IE se nos muestre que la intuición, el ser colaborador o empático, significa también que somos inteligentes. Cada uno de nosotros reconoce en sí mismo alguna de esas cualidades y otras: recordamos haber conversado en el recreo con un compañero que se veía triste y "soplar" o haber sugerido a alguien, durante un examen, una respuesta. Hemos organizado o hemos sido invitados a una fiesta, un paseo, una conversación o un juego. Somos, efectivamente, más inteligentes de lo que el colegio cree. Intuitivamente siempre hemos sabido que esas cosas tenían que ver, es decir, que lo emotivo y lo cognitivo en cada uno de nosotros conforman una unidad. La separación entre nuestra manera de abordar la socialización, de combinar el colegio con los líos del hogar, tenía que ver con la idea de nosotros como personas inteligentes, eficaces y en permanente construcción. Sin embargo, el colegio no enviaba ninguna señal "oficial" de que así fuera y nos quedábamos dudando acerca de que si lo que sucedía en los pasillos, en los recreos, en la cancha de fútbol, en la discoteca, fuera de las cuatro paredes del aula o de la dinámica de la clase, tuviera que ver con nuestra inteligencia o estuviera ligado a nuestro éxito o fracaso académico. Es como si todo lo anterior no tuviera que ver con el ámbito cognitivo. Nadie imaginaba entonces, que nuestras competencias sociales demostradas fuera de los espacios escolares, pudieran explicarse como expresión de nuestra inteligencia, porque los marcos conceptuales tradicionales de ese momento, así como las metodologías y técnicas que se derivaban de aquellos, simplemente no integraban, de manera importante, categorías del ámbito emocional como parte de la inteligencia.

La IE se considera como un conjunto de aptitudes que tiene en cuenta que el mundo de los sentimientos y de la razón, forman un conjunto inseparable en la realidad de un ser humano (Brockert y Braun, 1996).

La capacidad de comprensión de los problemas ajenos, la de comunicarnos con claridad, de trabajar en equipo, son algunas de las facetas que describen a una persona potencialmente útil a los demás, a la sociedad, una persona más equilibrada, que está más cerca que otras de su bienestar emocional.

Recordemos que la palabra emoción deriva su etimología de la raíz latina movere y el prefijo ex, que significan "mover hacia fuera" y la inteligencia, definida con Brockert y Braun (1996), se coloca en la conexión que existe entre las capacidades y necesidades el individuo y las demandas del mundo exterior. Por lo tanto, esas capacidades de percibir, comunicar, cooperar son parte de la inteligencia junto con otras. La inteligencia es la aptitud para asimilar la información, lo que en la visión tradicional, constituía un ámbito especifico de sabios o eruditos alejados de la realidad. Al revés, estos autores explican que la inteligencia “...no es ningún deporte de alta competición intelectual. Con ella se logra 
la asimilación de las informaciones que diariamente se presentan en la vida cotidiana y deben ser procesadas de manera rápida, espontánea y sin largas cavilaciones". (Brockert y Braun, 1996: p. 09).

Los mismos autores están convencidos de que la inteligencia emocional es más valiosa para triunfar en la vida que las nociones aprendidas en el colegio o en la universidad; creen que nuestro mundo, para progresar, requiere gente con alto coeficiente de inteligencia emocional más que genios, y que la convivencia de las personas en la familia y en la sociedad puede mejorar de manera evidente con un buen manejo de ese complejo de cualidades.

En cuanto a la teoría de las inteligencias múltiples, Antunes y Gardner (1996) destacan la eliminación de la idea preconcebida de la "inteligencia" única o general y combaten la de que se trate de un elemento neurológico aislado, independiente del ambiente. En este punto, las dos vertientes teóricas de la inteligencia emocional y las inteligencias múltiples podrían encontrarse y dialogar. Así, Gardner ha tratado de delinear la existencia de zonas definidas en el cerebro humano que corresponden a determinados espacios de cognición. Cada una de las zonas específicas podría expresar una forma diferente de inteligencia al ser responsable de un ámbito de funcionamiento específico en la solución de problemas o en la realización de producciones específicas, cada una válida para una cultura dada. En concordancia con lo anterior, el autor mencionado indica ocho criterios que hacen que una competencia pueda conceptualizarse como una inteligencia.

El primero es la identificación de la morada cerebral, es decir, la localización cerebral que se comprueba con la pérdida de esa capacidad a raíz de un daño físico en la corteza. El segundo es el criterio que deriva de los así llamados idiots savants, o sea, personas que dominan de manera excepcional un área del conocimiento, en un contexto de discapacidad en el que han desarrollado destrezas particulares, pero muestran dificultades para resolver problemas de índole distinta a aquella que dominan.

Otro criterio se basa en la hipótesis de que la inteligencia en las personas podría "dispararse" momentáneamente a partir de un estímulo que actuara a modo de "gatillo neuronal". El ejemplo más común es la sensibilidad especial de un niño ante la música.

Este criterio se complementa con el siguiente, el cuarto, que investiga la susceptibilidad a la modificación de la inteligencia, mediante entrenamiento, con la convicción de que las inteligencias no nacen "hechas" o completas y que el desarrollo de la humanidad ha influenciado su desarrollo y consolidación.

El quinto criterio se basa en la posibilidad de seguir la pista, en sentido evolutivo, a algunas inteligencias específicas o rasgos, mientras que el sexto se 
refiere a las investigaciones o pruebas particulares como las que se han realizado para la memoria.

El séptimo criterio hace referencia a las pruebas clásicas de la inteligencia, las cuales evidencian en la presencia de áreas específicas de su desarrollo como la lingüística o la matemática. Por último, Gardner pone como prueba de la existencia de ámbitos de desarrollo de la inteligencia, la creación por parte de los humanos de códigos específicos, como lo son, por ejemplo, las letras del alfabeto o la notación musical.

Será útil recordar brevemente que existe una polémica que opone el tema de las inteligencias múltiples al de la inteligencia emocional. Al respecto podemos destacar que para Gardner existe una inteligencia interpersonal, otra intrapersonal y además una "media inteligencia" que define como espiritual y que corresponde a la de los grandes místicos y que se expresa como la capacidad de entregarse al cuidado de un proyecto colectivo, poniéndolo por encima de los intereses personales. El concepto de inteligencia emocional de Goleman, en cambio, posee una intención divulgativa y se construye a partir de una recopilación de investigaciones y una postura epistemológica ligada a la psicología transpersonalista y a aportes conductistas.

Otro asunto que resulta útil vincular con el concepto de inteligencia emocional es el de creatividad (Brockert y Braun, 1996). El significado actual que se le ha dado al término "creatividad" se ha acuñado a partir de los años sesentas, durante los cuales la guerra fría y la competencia espacial entre los Estados Unidos y la entonces Unión Soviética requirieron la detección y formación de jóvenes científicos que pensaran de forma novedosa y divergente. El estudio de la creatividad, por lo tanto responde a exigencias educativas y formativas para asegurar la "producción" de estudiantes más creativos.

En ese mismo sentido, el concepto de creatividad puede entenderse como "pensamiento divergente", se trata de la capacidad de pensar lo que no se ha pensado hasta el momento, de inventar lo nuevo a partir de esfuerzos de síntesis y de la aplicación de la intuición.

Los sistemas educativos norteamericanos y soviéticos se revisaron de forma autocrítica en los años de guerra fría, ante la necesidad de fomentar en los estudiantes la creatividad, necesaria para ganar la competencia técnico-científica. Probablemente, la misma revisión tendría sentido llevarla a cabo ahora, en un momento histórico en el que, a la par de investigadores metódicos, motivados y dedicados, necesitamos a profesionales creativos en todas las disciplinas.

Tenemos la preocupación de que, en general, las capacidades que de veras la sociedad, la industria, el comercio y el sistema de las comunicaciones necesitan, se fomentan muy poco en el sistema educativo formal. Particularmente en el colegio y considerando las características del período de la adolescencia, hay 
cinco habilidades específicas que merecen ser potenciadas en el proceso educativo y estas son: la autoconciencia, el autocontrol, la motivación, la empatía y la habilidad social (Brockert y Braun, 1996).

La autoconciencia a veces se da por sentada: no siempre nos conocemos de verdad, ni reflexionamos acerca de nuestros sentimientos, capacidades y reacciones. Lo anterior es especialmente destacable cuando nos referimos a jóvenes en la edad de colegio. La adolescencia se caracteriza por la exploración de sí mismo con la consecuente colocación en el mundo social, en los diferentes escenarios a los cuales el adolescente se asoma (el grupo de pares, la familia, el colegio, el mundo del trabajo, los grupos informales, etc.).

Por su parte la autoconciencia es necesaria para ejercer de manera eficaz el autocontrol. Este último marca una medida de éxito en la vida (Beauvois y Dubois /en Moscovici, 1996) recuerdan como existen personas "externas" y personas "internas", en la tradición de los estudios sobre el locus of control. Las personas externas son las que tienden a poner al exterior de ellas las razones del éxito, de la suerte, de las victorias y derrotas de la vida. Las "internas" tienden a poner la atribución causal de los que les pasa en sí mismas. Estas últimas son, por lo general, las más exitosas. Eso no es una casualidad: se cree que cada sociedad construye los modelos de persona que mejor se puedan insertar en ella. Hoy en día ese modelo de persona, es la persona "interna", por lo tanto, en dominio de sí mismas en todos los sentidos.

En cuanto a la motivación, se trata de un término que describe quien es fiel a la tarea, no se deja desalentar por el fracaso y se dedica de manera constante a su objetivo. Así el autoconocimiento, el autocontrol y la motivación representan tres dimensiones de la inteligencia emocional ligadas al mundo interno. Los dos aspectos que siguen tienen más relación con el mundo social: tres más hacia lo interno y dos hacia lo exterior: la empatía y las habilidades sociales. La empatía es la capacidad de ponerse en los zapatos del otro, entender intuitivamente lo que siente: unas características de las personas que deriva y a la vez, potencia al autoconocimiento, permite entender al otro, anticipar sus acciones, negociar y comunicar más fácilmente.

La vida no es solo explorarse a uno mismo: también se trata de hacer algo con los demás y es aquí donde entran a jugar las habilidades sociales que consisten en orientarse hacia las demás personas con energía. Los últimos aspectos tratados muestran evidentemente su parentesco con los conceptos de inteligencia inter e intrapersonal en la teoría de las inteligencias múltiples de Gardner.

Creemos que se trata de vertientes que el colegio puede aprovechar, una vez que se tengan claros los conceptos, de manera práctica y económica a la vez. 
Las cinco habilidades se vinculan con la inteligencia emocional y con la creatividad en cuanto base para su fomento.

Se presentarán a continuación algunas consideraciones finales a manera de conclusiones.

1. Los docentes de secundaria, y nos atreveríamos a afirmar que los de primaria también, nos encontramos en la actualidad frente a nuevas situaciones educativas que no siempre sabemos cómo resolver con éxito. Las características de dichas situaciones, presentan aristas nuevas, que no imaginábamos, y ante las cuales, nos hace falta posicionarnos para construir abordajes y respuestas creativas y hasta valientes, que alivien la sensación de frustración, soledad y pérdida de sentido de nuestra práctica.

Esas situaciones incluyen y permean la totalidad del currículo, desde el marco político económico de la globalización y su correlato cultural del posmodernismo, que generan condiciones particulares de socialización fuertemente influenciadas por contextos de información que no es neutra, sino que sirve a los intereses del mercado, las cuales perfilan las formas de sentir, pensar y actuar de nuestros jóvenes, hasta las vivencias en el aula, la mediación pedagógica, los recursos didácticos, entre otros. Esto dibuja un paisaje cctidiano del colegio dinámico, cambiante, diferente de aquél para el que fuimos, en general, formados como educadores. Así lo expresa el pedagogo de la Universidad Internacional de Andalucía, en España, Ángel Pérez (2003):

...la escuela contemporánea que estamos viviendo, en su forma organizativa del espacio, del tiempo y del currículo, es una escuela decimonónica, es una escuela premoderna, que ha cambiado muy poco desde la época de su pretendida universalización, hasta nuestros días. Los docentes que estamos trabajando en esa escuela, por otro lado, hemos sido educados, hemos sido formados en la racionalidad moderna, en el espíritu de la Revolución Francesa, y los alumnos y las alumnas, con las que hemos de trabajar, se están formando, se están desarrollando y están construyendo sus esquemas de pensamiento, de sentido y de conducta, en una época postmoderna.

Por ello, es general el sentimiento de incertidumbre y desconcierto, que nos obliga a readecuar nuestras estrategias, a reinterpretar las teorías, e identificar en ellas caminos posibles para la comprensión y solución de problemas de la realidad en la que viven los estudiantes. 
En esta nueva y difícil tarea, resultan fundamentales los aportes de las investigaciones que se desarrollan en diversas áreas del conocimiento, como es el caso de las teorías sobre la inteligencia, porque ayudan a la comprensión de los cambios, de la complejidad de las dinámicas que construyen nuevos escenarios y constituyen plataformas teóricas desde donde es posible proponer, críticamente, nuevas formas de enseñar y aprender aquello que resulte necesario para potenciar en los estudiantes y en nosotros mismos, la construcción de nuestros proyectos de vida como personas y como sociedad. Mediante los aportes teóricos de las investigaciones como a la que hemos hecho referencia en este artículo, cambian los lentes conceptuales, se mueven los prismas del conocimiento y, como un caleidoscopio; surgen las vías, las formas y colores, los nuevos textos, signos y símbolos, lenguajes para explicar, comprender y educar mejor. Porque en esta intrincada red de informaciones y comunicaciones en las que estamos sumergidos, los cambios y propuestas en un sitio, influyen, tarde o temprano, en las demás áreas del conocimiento. Así, los avances en el estudio de la medicina, la psicología, la filosofía y el arte, por citar tan solo unas cuantas, provocan cambios en la pedagogía y mediante el trabajo docente, impactan las formas de pensamiento de las nuevas generaciones, las cuales, a su vez, reinterpretan sus aprendizajes y modifican sus vidas cotidianas, comunicando soluciones creativas, felices e innovadoras a los nuevos y tal vez hasta a los viejos problemas.

2. Lo anterior puede resultar coherente en términos de conocimiento declarativo, verbal, pero, ¿cómo llevarlo a nuestras prácticas pedagógicas? Para empezar, sabemos que nada negaría tanto la naturaleza de las teorías acerca de las inteligencias, y especialmente de la inteligencia emocional, que la elaboración de actividades de enseñanza y aprendizaje construidas en el vacío, es decir, sin vinculación con los contextos de aula en la que se aplicarían y desconociendo las situaciones de los docentes y las de los estudiantes, sus concepciones sobre la educación, la estructura de los conocimientos y la disciplina que se pretenden enseñar, los valores que conciernen a tales actividades pedagógicas, así como la intencionalidad que orientaría el trabajo.

Creemos que más que una lista de actividades didácticas, la aplicación de la inteligencia emocional en el trabajo de aula, requiere de cambios en las concepciones acerca de las tareas educativas de los docentes de secundaria, que los lleven a resignificarlas en relación con los nuevos retos y responsabilidades éticas que plantean los problemas de la sociedad contemporánea, que están muy lejos de la preparación de los estudiantes para dar las pruebas nacionales. Tal reconceptualización, demanda la construcción de espacios para la reflexión colectiva, tan escasos en la realidad cotidiana del trabajo 
docente en secundaria en este país. El estudio y la reflexión acerca de temas como la inteligencia emocional, favorece los cambios conceptuales que se reflejarían, a su vez, en cambios actitudinales y procedimentales por parte de los docentes y es posible que con ello, se logren mejores ambientes de confianza, dialógicos, respetuosos de las diferencias, democráticos, inclusivos y equitativos, que potencien los aprendizajes significativos en los estudiantes, porque están vinculados con sus vivencias, les ayudan a pensar y a aprender mejor, de esta manera, fortalecen su bienestar y su desarrollo personal y social.

\section{Referencias}

Antunes. C. (1998). Estimular las inteligencias múltiples. ¿Qué.son y cómo se manifiestan? Madrid: Narcea ediciones.

Alvarado. M. (2000). Inteligencia emocional y resiliencia. Tesis para optar al título de Licenciatura en Psicología. Universidad de Costa Rica. San José, Costa Rica.

Beauvois. J.L. y Dubois. N. (1994). Credenze interne e credenze esterne. En: Moscovici, cit.

Bertolini, P. (2003). Educazione e politica. Milano: Raffaello Cortina Editore.

Brockert. S. y Braun. G. (1996). Los test de la inteligencia emocional. Barcelona: Robin Book.

Consejo Nacional de Rectores, Consejo de Planificación de la Educación Superior, OPES 13(/2003). Propuestas: Primer Congreso Nacional de Educación, Por una educación Renovada. Uladislao Gámez Solano. San José: Publicaciones 2003.

D’Antoni, M. Y Pacheco, X. (2003). "El 11 de septiembre desde una mirada adolescente". I Congreso Nacional de educación. Por una educación renovada. San José, Costa Rica. En: Consejo Nacional de Rectores.

Gardner, H. (1996). Estructuras de la mente. México, D. F.: Fondo de Cultura Económica. 
Goleman, D. (1995). Emocional inteligente. Why it can matter more than IQ. New York: Bantam Books.

Groterberg. E. (2000). www.mentahelath.org/special/schoolviolence/ resilence.htm

Mc Laren, P. (1986). La escuela como un performance ritual. Hacia una economía política de los símbolos. México, D.F.: Siglo XXI Editores.

Moscovici, S. (curador). (1994). La relazione con L altro. Milano: Raffaello Cortina Editore.

Pérez Gómez, Ángel (2003). "La nueva escuela en la era de la información”. I Congreso Nacional de Educación Por una educación renovada, San José: Consejo Nacional de Rectores.

Varios autores, (1998). Manual para la identificación y la promoción de la resilencia en los niños y adolescentes. Fundación Kellog. 